\title{
The Functional Impact of the Intrastriatal Dopamine Neuron Grafts in Parkinsonian Rats Is Reduced with Advancing Disease
}

\author{
Nathalie Breysse, ${ }^{1,2}$ Thomas Carlsson, ${ }^{1}$ Christian Winkler, ${ }^{1,3}$ Anders Björklund, ${ }^{2}$ and Deniz Kirik ${ }^{1}$ \\ ${ }^{1}$ Central Nervous System Disease Modeling Unit and ${ }^{2}$ Neurobiology Unit, Section for Neuroscience, Department of Experimental Medical Science, Lund \\ University, 22184 Lund, Sweden, and 3 Department of Neurology, Hannover Medical School, 30625 Hannover, Germany
}

\begin{abstract}
Clinical trials involving intrastriatal transplants of human embryonic mesencephalic tissue have provided proof-of-principle that nigral dopamine (DA) neurons can survive and functionally integrate into the host neural circuitry. However, the degree of graft-induced symptomatic relief differs significantly between the patients. This variability has led to investigations aimed at identifying factors that could affect the clinical outcome. The extent and pattern of dopaminergic denervation in the brain may be one of the major determinants of the functional outcome after intrastriatal DA cell grafts. Here, we report that in animals subjected to an intrastriatal 6-hydroxydopamine lesion of the striatal dopaminergic afferent, the integrity of the host dopaminergic innervation outside the areas innervated by the graft is critical for optimal function of DA neurons placed in the striatum. Established graft-induced functional recovery, as assessed in the stepping and cylinder tests, was compromised in animals in which the dopaminergic lesion was extended to include also the medial and ventral striatum as well as the cortical and limbic DA projections. Poor clinical outcome after transplantation may, thus, at least in part, be caused by dopaminergic denervation in areas outside the graft-innervated territories, and similarly beneficial effects initially observed in patients may regress if the degeneration of the host extrastriatal DA projection systems proceeds with advancing disease. This would have two implications: first, patients with advanced disease involving the ventral striatum and/or nonstriatal DA projections would be unlikely to respond well to intrastriatal DA grafts and, second, to retain the full benefit of the grafts, progression of the disease should be avoided by, for example, combining cell therapy with a neuroprotective approach.
\end{abstract}

Key words: Parkinson's disease; cell transplantation; dopamine; functional recovery; motor behavior; stereology

\section{Introduction}

Parkinson's disease (PD) is the second most common neurodegenerative disorder, with a prevalence of $1 \%$ of the global population. The main underlying pathology is a progressive loss of dopamine (DA) synthesizing neurons located in the ventral mesencephalon (VM), particularly the cells located in the substantia nigra (SN) pars compacta. This leads to a dramatic decrease in the dopaminergic innervation of the striatum (Nyberg et al., 1983; Kish et al., 1988; Fearnley and Lees, 1991). The neurodegeneration is typically prominent within the ventral tier of the $\mathrm{SN}$ in the early stages of the disease. Other dopamine-containing cell groups such as the dorsal tier, the ventral tegmental area (VTA), and the perirubral and retrorubral areas, constituting the mesocorticolimbic dopaminergic system, are usually affected to a

Received Feb. 12, 2007; revised April 16, 2007; accepted April 17, 2007

This work was supported by Swedish Research Council Grants K2003-33SX-14552-018, K2003-33P-14788-018, K2005-33IT-15332-018, and K2005-33X-14552-03A. N.B. was a postdoctoral fellow supported by the Marie Curie Intra-European Exchange Program within the 6th Framework Program of the European Union. We thank Ulla Jarl, Anneli Josefsson, and Bengt Mattsson for their expert technical support.

Correspondence should be addressed to Dr. Deniz Kirik, Wallenberg Neuroscience Center, Biomedical Center A11, 22184 Lund, Sweden. E-mail: Deniz.Kirik@med.lu.se.

N. Breysse's present address: NPS Pharmaceuticals, 101 College Street, South Tower, Suite 800, Toronto, Ontario, Canada M5G $1 \mathrm{~L} 8$

D01:10.1523/JNEUROSCI.0626-07.2007

Copyright $\odot 2007$ Society for Neuroscience $\quad$ 0270-6474/07/275849-08\$15.00/0 lesser extent, whereas the central gray substance may remain nearly intact. In some moderate-to-severe Parkinson's disease cases, however, neurodegeneration is more widespread and will involve also the dorsal tier neurons and the VTA (Hirsch et al., 1988; Damier et al., 1999).

The neural transplantation strategy has been based on the idea that fetal DA neurons can be used to replace the nigral DA cells lost in patients with PD. Clinical trials using transplants of human embryonic mesencephalic tissue in PD patients have revealed that grafted DA neurons can survive, appropriately differentiate and reinnervate the striatum, release DA, and become functionally integrated into the host neural circuitries (Lindvall et al., 1990; Kordower et al., 1995; Piccini et al., 1999; Mendez et al., 2005). However, the magnitude of transplant-induced functional outcome has been variable with some patients displaying major improvements and others no or only minor clinical response (Peschanski et al., 1994; Hagell et al., 1999; Hauser et al., 1999; Brundin et al., 2000; Mendez et al., 2000; Freed et al., 2001; Cochen et al., 2003; Olanow et al., 2003) [for detailed review of the literature, see Winkler et al. (2005)]. Importantly, the extent of DA neurodegeneration and the preoperative distribution and magnitude of loss of DA innervation in the forebrain have not been so far taken into consideration, neither in the selection of patients for grafting nor in the design of the transplant procedure. 
Interestingly, studies using selective nigrostriatal DA lesions suggest that good functional outcome is obtained in animals with limited dopaminergic denervation outside the striatal area innervated by the graft. In contrast, animals with complete lesions of the ascending DA projection system showed poor or no improvement in spontaneous motor behaviors, suggesting that the extent of DA denervation in regions outside the graft-innervated area may be essential for the full beneficial effects of intraputaminal DA cell replacement (Winkler et al., 1999, 2005; Kirik et al., 2001). This hypothesis is supported by previous observations in grafted PD patients obtained by 18-fluorine-L-3,4-dihydroxyphenylalanine $\left({ }^{18} \mathrm{~F}\right.$-DOPA) and ${ }^{11} \mathrm{C}$-raclopride positron emission tomography (PET) (Piccini et al., 2005). Indeed, those patients who exhibited the best global outcome score showed increased putaminal ${ }^{18} \mathrm{~F}$-DOPA uptake after transplantation without any significant reduction in the nongrafted ventral striatum. In contrast, in patients with modest or poor symptomatic relief, despite similar graft-induced increases in putaminal uptake, the ${ }^{18} \mathrm{~F}$-DOPA signal in the ventral striatum (area not innervated by the graft) was markedly reduced.

Here, we designed an experiment to directly test the hypothesis that the functional impact of intrastriatal VM grafts could be compromised by advancing disease-causing loss of DA innervation in areas not innervated by the grafts. The study was planned to first establish multideposit grafts providing widespread dopaminergic fiber innervation to the caudate-putamen and good behavioral effects, and then ask whether extension of the lesion to involve the remaining host forebrain DA projections would compromise the functional impact of these grafts.

\section{Materials and Methods \\ Animals and surgical procedures}

One hundred adult female Sprague Dawley rats were used in this study (B\&K Universal, Stockholm, Sweden). The animals weighing 225-250 g in the beginning of the study were housed under $12 \mathrm{~h}$ light/dark cycle with ad libitum access to water and food. Surgery for 6-hydroxydopamine (6OHDA), lesions and transplantation of fetal cells were conducted under Hypnorm and Dormicum (both from Apoteksbolaget, Stockholm, Sweden) anesthesia using a $5 \mu \mathrm{l}$ Hamilton syringe fitted with a glass capillary (outer diameter of $60-80 \mu \mathrm{m}$ ) attached to a stereotaxic frame (Stoelting, Wood Dale, IL). All surgical procedures and behavioral testing were conducted in accordance with the regulations set by the Ethical Committee for use of Laboratory animals in the Lund-Malmö Region.

\section{Experimental design}

In the beginning of the experiment, all animals received a unilateral 6-OHDA lesion in the striatum (see Fig. 1). Animals with severe lesions of the nigrostriatal pathway were selected based on a behavioral screen using amphetamine-induced rotation and cylinder tests. Of the one hundred lesioned animals, 77 exhibited $\geq 6$ full body turns/min toward the side of lesion in the drug-induced rotation test and, of those, 55 also exhibited impairments in paw-use in the cylinder test (defined here as $\leq 25 \%$ left paw touches) and forelimb placement in the stepping test ( $\leq 2$ steps performed with the left forelimb in the forehand direction). Thirtyfive rats fulfilling all behavioral criteria were finally included in the experiment and, thus, were allocated into one of two balanced groups. Eight weeks after the intrastriatal lesion, one of the groups received transplantation of $\sim 450,000$ fetal VM cells distributed over 15 deposits in the lesioned striatum $(n=16)$, whereas the other group was kept as nontransplanted group $(n=19)$. Twenty-three weeks after the transplantation surgery, a second 6-OHDA lesion was performed in the medial forebrain bundle (MFB) on one-half of the nongrafted control animals (group $2 ; n=10$ ) and one-half of the grafted animals (group $4 ; n=9$ ). Whereas, in contrast, the remaining animals were followed as striatal lesion groups (group 1, $n=9$; group 3, $n=7$ ). During the time course of the study, a battery of behavioral tests including the cylinder, stepping, and the amphetamine-induced rotation tests were conducted at 4-7, 25-28, and 36-39 weeks after the lesion (Fig. 1, test sessions 1-3). Finally, the experiment was terminated at 42 weeks after the first 6-OHDA lesion (i.e., 34 weeks postgrafting) and the animals were killed by paraformaldehyde perfusion for histological and morphological analysis.

\section{Surgical procedures for lesion of the ascending DA \\ projection systems}

The initial lesion were performed by unilateral injections of a total of 28 $\mu \mathrm{g}$ of 6-OHDA [3.5 $\mu \mathrm{g} / \mu \mathrm{l}$ (calculated as free base) in $0.2 \mathrm{mg} / \mathrm{ml}$ ascorbic acid in saline; Sigma, Stockholm, Sweden], distributed over four sites in the striatum to obtain a strong partial DA denervation. The coordinates, calculated from the bregma using the rat brain atlas (Paxinos and Watson, 1986), for the anteroposterior (AP) and the mediolateral (ML) positions were as follows: (1) AP, +1.3; ML, -2.6; (2) AP, +0.4; ML, -3.2 ; (3) AP, -0.4 ; ML, -4.2 ; (4) AP, -1.3 ; ML, -4.5 . The dorsoventral (DV) position of all injections was $-5.0 \mathrm{~mm}$ below the dura and the tooth bar was set to 0.0 . In this lesion paradigm, a glass capillary (outer diameter of $60-80 \mu \mathrm{m}$ ) was fitted on the microinjection needle to ensure minimal mechanical damage caused by the multiple injection procedure.

A second 6-OHDA lesion was conducted on half of the animals and targeted to the MFB. These animals received two injections of 7 and 8.75 $\mu \mathrm{g}$ of 6-OHDA (3.5 $\mu \mathrm{g} / \mu \mathrm{l}$, as above), respectively, at the following coordinates, calculated relative to the bregma: (1) AP, -4.4 ; ML, - 1.2; (2) $\mathrm{AP},-4.0 ; \mathrm{ML},-0.8$. The DV positions for the two sites were -7.8 and $-8.0 \mathrm{~mm}$ below the dura and the tooth bar was set at -2.4 and $+3.4 \mathrm{~mm}$, respectively. All of the 6-OHDA injections were infused at a rate of 1 $\mu \mathrm{l} / \mathrm{min}$ and the needle was left in the brain for an additional $3 \mathrm{~min}$ before it was slowly retracted.

\section{Cell suspension preparation and transplantation surgery}

A single cell suspension from fetal VM tissue pieces was prepared after the procedures described previously (Nikkhah et al., 1994). Briefly, tissue from the VM of 14-d-old rat embryos was dissected and incubated for 30 $\min$ at $37^{\circ} \mathrm{C}$ in $0.1 \%$ trypsin and $0.05 \%$ DNase in DMEM. The tissue was then mechanically dissociated into a single-cell suspension and concentrated by centrifugation. The pellet was further resuspended in DMEM to $\sim 100,000$ cells/ $\mu$ l with a viability of $>99 \%$ at the beginning of the grafting session. A total number of 450,000 cells were distributed over five sites in the 6-OHDA-lesioned striatum at the following coordinates relative to the bregma: (1) AP, $+1.1 \mathrm{~mm}$; ML, $-3.4 \mathrm{~mm}$; (2) AP, $+0.6 \mathrm{~mm}$; $\mathrm{ML},-2.5 \mathrm{~mm}$; (3) AP, $+0.3 \mathrm{~mm}$; ML, $-3.8 \mathrm{~mm}$; (4) AP, $-0.5 \mathrm{~mm}$; ML, $-4.2 \mathrm{~mm}$; (5) AP, $-1.5 \mathrm{~mm}$; ML, $-4.5 \mathrm{~mm}$. For each site, three deposits of $0.3 \mu \mathrm{l}$ were placed at the DV coordinates $-5.3,-4.3$, and -3.3 , and the tooth bar was set to $-3.3 \mathrm{~mm}$. The viability of the remaining suspension after the transplantation surgery was estimated to $95-98 \%$. A total number of 16 animals received cell suspension in the striatum (groups 2 and 4), whereas 19 animals were kept as sham-operated controls (groups 1 and 3 ).

\section{Behavioral analysis}

Amphetamine-induced rotation. Testing was performed in automated rotational bowls (AccuScan Instruments, Columbus, $\mathrm{OH}$ ) as described previously (Ungerstedt and Arbuthnott, 1970). At 4 weeks after the striatal 6-OHDA lesion, at 17 weeks after the transplantation surgery, and at 8 weeks after the second 6-OHDA lesion, right and left body turning were recorded over $90 \mathrm{~min}$ after an intraperitoneal injection of $2.5 \mathrm{mg} / \mathrm{kg}$ D-amphetamine sulfate (Apoteksbolaget). The data are expressed as net full body turns per min where rotation toward the side of the lesion was given a positive value.

Cylinder test. The cylinder test was conducted as described previously to assess the animals' spontaneous forelimb use (Schallert and Tillerson, 1999; Kirik et al., 2001). This test is aimed at scoring the weight-shifting movements of the forelimbs during spontaneous exploratory behavior. For this purpose, the animal were individually placed in a glass cylinder (diameter, $20 \mathrm{~cm}$ ) in which they could move freely while being videotaped. An observer blinded to the identity of the animals scored the videotapes and noted for each animal a total of 20 contacts made by the left and right forepaw with the wall of the cylinder. The data from this test is presented as left (impaired) paw use as a percentage of the total number 
of touches, where a symmetric paw use in an unbiased animal would receive a score of $50 \%$.

Stepping test. To measure the forelimb akinesia, a modified version of the stepping test was performed as described previously (Schallert et al., 1992; Olsson et al., 1995; Kirik et al., 1998). Briefly, the investigator firmly held the animal's hindlimbs and one of the forelimbs, although letting the unrestrained forepaw contact the surface of the examination table. The rats were then moved sideways $(\sim 0.9 \mathrm{~m}$ in $5 \mathrm{~s})$ across the surface while the experimenter counted the number of adjusting steps performed in the forehand and backhand directions separately. Five to 7 weeks after the first 6-OHDA lesion, the animals were habituated to the handling procedure for a week and further tested three times daily for 3 consecutive days to reach a stable baseline performance. This test was also performed at 20 weeks after the transplantation surgery, and at 5 weeks after the second 6-OHDA lesion. The data are expressed as the average number of forepaw adjustment steps for the impaired (left) paw in the forehand direction collected at 3 consecutive testing days per test session.

\section{Histological analysis}

At the end of the experiment, at 34 weeks after the transplantation surgery, the animals were deeply anesthetized with sodium pentobarbital (Apoteksbolaget) and transcardially perfused with $50 \mathrm{ml}$ of physiological saline followed by $250 \mathrm{ml}$ of ice-cold paraformaldehyde in $0.1 \mathrm{M}$ phosphate buffer, $\mathrm{pH} 7.4$, over $5 \mathrm{~min}$. Thereafter the brains were removed, postfixed for additional $3-4 \mathrm{~h}$ in the same fixative and further transferred in to $25 \%$ sucrose for cryoprotection. The tissue was cut at $35 \mu \mathrm{m}$ thickness in six series using a freezing slide microtome (SM2000R; Leica, Oberkochen, Germany).

Immunohistochemistry was performed to visualize the TH-positive cell bodies and their fiber terminals in the midbrain and in the grafted striatum. Briefly, free-floating sections were quenched for $10 \mathrm{~min}$ in potassium phosphate buffer (KPBS) containing $3 \% \mathrm{H}_{2} \mathrm{O}_{2}$ and $10 \%$ methanol. The sections were then preincubated for $1 \mathrm{~h}$ in $5 \%$ normal horse serum plus $0.25 \%$ Triton X-100 in KPBS, and further by over-night incubation in room temperature with a primary antibody recognizing the TH protein (MAB 318, mouse IgG, 1:4000; Millipore, Temecula, $\mathrm{CA})$. The second day started with a $1 \mathrm{~h}$ incubation with a biotinylated secondary antibody (BA 2001, horse anti-mouse, 1:200; Vector Laboratories, Burlingame, CA), which was followed by an additional $1 \mathrm{~h}$ incubation in avidin-biotin-peroxidase solution (ABC Elite; Vector Laboratories). Finally, visualization of the bound antibody was done using the chromogen $3,3^{\prime}$-diaminobenzidine and $0.01 \% \mathrm{H}_{2} \mathrm{O}_{2}$. The sections were then mounted on chrome-alum-coated glass slides, dehydrated in escalating alcohol concentration cleared in xylene, and coverslipped using Depex.

\section{Morphometric analysis}

Stereological cell counting of TH-positive cells in the SN and the grafted striatum. Estimation of the total TH-positive cell number in the $\mathrm{SN}$, in the VTA, and the grafted striatum were performed using a $40 \times 1.30$ oil objective with the Olympus (Albertslund, Denmark) CAST system version 2.0. Briefly, the structure of interest was delineated and a counting frame was randomly placed on the first counting area, which was then systematically moved throughout the inclusion area (Kirik et al., 1998, 2001). The penetration of the antibody was determined by registration of the depth of each counted neuronal profile that appeared in focus within the counting frame. This analysis revealed an incomplete penetration of antibody leaving $\sim 6-8 \mu \mathrm{m}$ in the center poorly stained (Torres et al., 2006). The inclusion volume estimating total number of cells was therefore calculated excluding this portion of the sections, and was done according to the optical fractionator principle as described (West, 1999). The SN was delineated from the rostral tip of the pars compacta to the caudal end of the pars reticulata. A vertical line passing through the medial tip of the cerebral peduncle and the medial terminal nucleus of the accessory nucleus of the optic tract was used as the medial border. The VTA was delineated using the medial terminal nucleus of the accessory nucleus of the optic tract as the lateral border and the midline as medial border. The dorsal tips of the mamillary and the interfasicular nuclei defined the ventral border and dorsally the ventral tip of the red nucleus was used as inclusion border. Both structures were counted from six to nine equally spaced sections constituting one complete series.

TH-positive fiber density measurements. Measurements were performed using images of coronal sections that were captured using a digital camera (ProgRes C14; Jenoptik, Jena, Germany) and an illumination table at seven rostral-caudal levels, which corresponded to AP +1.60 , $+1.00,+0.20,-0.30,-0.90,-1.40$ and -2.10 , with respect to the bregma according to the rat stereotaxic atlas (Paxinos and Watson, 1986). The mean optical density of the TH-positive fiber staining in the striatum (levels 1-7) and nucleus accumbens (NAcb) (levels 1-2) was then measured using the Image J software version 1.33 for MacOSX platform (National Institutes of Health, Bethesda, MD; http://rsb.info.nih.gov/ij/). The striatum was included from the lateral ventricle to the external capsule and the border to the NAcb in the two anterior sections, a horizontal line connecting the ventral end of the ventricle via the anterior commissure to the external capsule in more caudal levels. The ventral border of the NAcb was marked dorsal to the ventral pallidum and the olfactory tubercle. Area covered by graft cores, where the cell bodies of the TH-positive neurons were located, was excluded from the delineation and nonspecific background was correlated by subtraction of the nonspecific binding as measured from the corpus callosum and completely denervated areas of the striatum. The data are expressed as a percentage of the intact side and represent the average of the seven levels for the striatum and two rostral sections for the NAcb, as described above.

\section{Statistical analysis}

Comparisons between groups were performed using one-way or twoway ANOVA, where appropriate. In cases in which the overall ANOVA indicated significant effects, post hoc analysis was performed using Tukey's honest significant difference analysis. Behavioral tests were analyzed using repeated-measures ANOVA followed by individual contrast post hoc analysis. All statistical analyses were performed using the JMP statistical software version 5.0.1.2 (SAS Institute, Cary, NC) and the statistical significance was set to $p<0.05$.

\section{Results}

\section{Behavioral impairments after intrastriatal dopamine depletion and the impact of VM transplants on motor function}

The present study was designed to demonstrate that the spared ascending DA projections in the host contribute to the motor functional improvements mediated by the intrastriatal DA cell grafts in rats with partial, intrastriatal 6-OHDA lesion. For this purpose, all of the animals included in the study first received a unilateral intrastriatal 6-OHDA lesion and were tested with a battery of behavioral assays consisting of amphetamine-induced rotation, cylinder, and stepping tests (Fig. 1, test session 1). In the pretransplant behavioral assessment, the animals showed variable impairments in motor function on the contralateral side to the lesion in the two spontaneous motor tests. Animals fulfilling the inclusion criteria (two or less steps in the stepping test and $\leq 25 \%$ impaired paw use in the cylinder test) were included in the study. We showed previously that these behavioral selection criteria successfully identify animals with severe intrastriatal DA lesions and that these animals do not display any spontaneous behavioral recovery, even when followed for several months after the lesions (Kirik et al., 2001).

Based on the initial behavioral screen, the animals were allocated to two groups balanced with respect to their behavioral test scores. Group comparisons for baseline behavioral measures were $F_{(1,34)}=0.62$ and $p=0.44$ for the stepping test, $F_{(1,34)}=$ 0.94 and $p=0.34$ for the cylinder test, and $F_{(1,34)}=0.14$ and $p=$ 0.71 for the amphetamine rotation test. One of the groups were then grafted with 15 small deposits of fetal VM tissue implanted along five injection tracks covering the entire head and tail of the 
striatal complex, whereas the other group received a sham operation. After a 6 month follow-up period and completion of a second set of behavioral tests (Fig. 1, test session 2), some of the animals in each arm received an injection of the 6-OHDA toxin in the MFB that was aimed to remove the remaining ascending midbrain DA projections spared by the first, intrastriatal 6-OHDA lesion.

The performance of the animals in each of the four groups was significantly affected by the intrastriatal 6-OHDA lesion, showing reduced contralateral forelimb use in the cylinder and the stepping tests $(8.9 \pm 3.2$ to $18.6 \pm 3.6 \%$ left paw use, and 0-1 steps, respectively) (Fig. $2 A, B)$, and high-level ipsilateral turning behavior in the amphetamine-induced rotation test $(12.9 \pm 1.6$ to $15.6 \pm 2.0$ turns/ min) (Fig. 2C). These deficits are consistent with a severe striatal dopaminergic loss. After transplantation, graft-induced functional improvement was observed in all three tests used (test session 2): in the stepping test, grafted animals (groups 2 and 4, respectively) (Fig. 1) improved to $5.4 \pm 1.1$ and $3.9 \pm 0.8$ steps at 4 months after transplantation (Fig. 2A) (repeated-measures ANOVA, $\left.F_{(3,31)}=13.32, p<0.0001\right)$. In the cylinder test, weightbearing wall touches increased to $74 \pm 5$ and $76 \pm 6 \%$ of the total paw use, respectively, suggesting preferential use of the contralateral paw (Fig. 2 B) (repeated-measures ANOVA, $F_{(3,31)}=64.35$, $p<0.0001)$. Finally, amphetamine-induced ipsilateral turning behavior was overcompensated (i.e., reversed to the contralateral direction; $-3.3 \pm 1.8$ and $-4.9 \pm 0.9$ turns/min; repeatedmeasures ANOVA, $F_{(3,31)}=53.47, p<0.0001$ ) (Fig. $2 C$ ). Overall, the nongrafted animals retained their ipsilateral bias in the rotation tests and remained poor in the paw use and forelimb placement tests.

\section{Effect of advancing disease on the functional impact of mature intrastriatal dopaminergic grafts}

In the third stage of the experiment, part of the VM-grafted animals and nongrafted controls were subjected to a second lesion to remove the dopaminergic projection fibers originating from the VTA and the medial parts of SN innervating mainly the prefrontal and cingulated cortex, NAcb, septum, and amygdala, as well as the most ventral and medial parts of the striatum (Fig. $3 A, E-H$ ). The partial lesion that was used in the first stage of the experiment is known to deplete dopaminergic fibers almost exclusively in the dorsal striatum and cause degeneration of cells preferentially in the SN pars compacta.

The impact of the second lesion was assessed starting 4 weeks after surgery (test session 3 ). Indeed, the second lesion applied in group 4 completely reversed the improvement in the stepping test induced by the intrastriatal transplants. The number of steps obtained with the contralateral paw was no longer different from the performance observed in the nongrafted control animals or the pregraft baseline scores (Fig. $2 A$ ). In the cylinder test, the second lesion partially, but significantly, reduced the use of the contralateral paw (Fig. $2 B$ ). In contrast, the improvement observed in turning behavior was unaffected, suggesting a lower requirement for recovery in this test (Fig. 2C).

\section{Effects of the partial and complete DA lesion on host DA projections}

The four-site intrastriatal lesion induced profound denervation of the lateral and caudal sectors of the caudate-putamen (Fig. $3 A$ ). Reduction of TH-positive fiber density was seen in the dorsal striatum throughout the rostrocaudal extent of the head of the caudate-putamen, as well as the entire caudal part including the ventral sector. Innervation of the NAcb, including the adjoining rostromedial part of the head of the caudate-putamen, was only marginally affected ( $<20 \%$ reduction compared with the control side) (Fig. $3 A, K, Z$ ). DA projections to nonstriatal areas (i.e., lateral septum and the olfactory tubercle) were almost completely spared (Fig. 3I,L). Extending the denervation by the second 6-OHDA lesion in the MFB induced a near-total loss of the initially spared DA projections (Fig. 3, compare $A, B$ ). This double lesion resulted in a widespread denervation of both striatal, and limbic and cortical areas (Fig. 3B, compare $I, M ; J, N ; K, O ; L, P$ ). Importantly, fiber densities measured in the nongrafted striatum in the lesion control groups (groups 1 and 2) was reduced from $\sim 20 \%$ of normal to $<10 \%$ (Fig. $3 Y$ ), whereas the reduction of TH-positive staining in the NAcb was reduced by $85-90 \%$ (Fig. 3Z).

The total number of TH-positive neurons in the $\mathrm{SN}$ and the VTA was assessed separately for each structure and for each side of the brain by stereological quantification methods. In the $\mathrm{SN}$, the total number of TH-positive neurons on the unlesioned control side varied between $8929 \pm 446$ and 11,919 \pm 655 (Fig. 4D). In the intrastriatal 6-OHDA-lesioned animals, TH-positive neurons in the SN were reduced by $78-80 \%$ ( $2467 \pm 361$ and $2472 \pm 357$, respectively in the two groups), whereas the VTA neurons were left intact (Fig. 4, compare $A$, $B$, quantified in $D, E)$. In the animals that received the second 6-OHDA lesion into the MFB (i.e., groups 2 and 4), only $\sim 1-2 \%$ of the TH-positive cells were left in the SN (120 \pm 23 and $125 \pm 19$, respectively in the two groups). In the VTA, the TH-positive cell loss induced by the intrastriatal 6-OHDA lesion was $<15 \%(9588 \pm 632$ and $10,359 \pm 1333$ on the lesion side, vs 10,589 \pm 435 and 12,385 \pm 981 on the intact side) (Fig. $4 E)$. After the second MFB lesion, the number of TH-positive cells in the VTA was reduced by $\sim 50 \%$ in the two lesioned groups ( $4721 \pm 263$ and $4773 \pm 239$ in groups 2 and 4 , respectively) (Fig. $4 E$ ). 
A

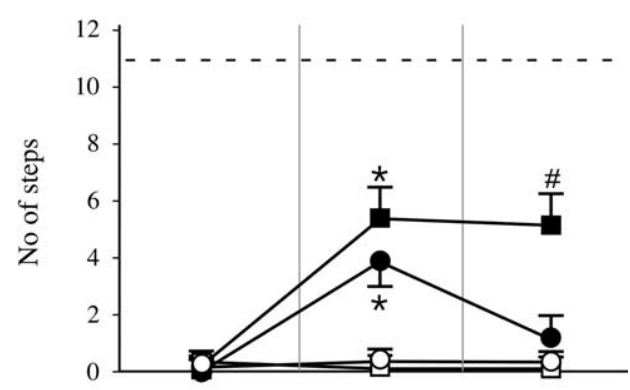

B Cylinder test

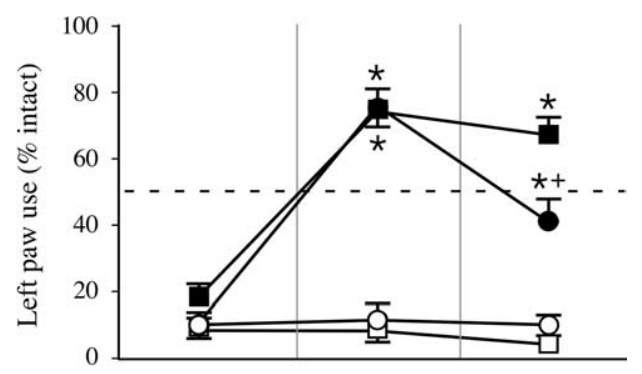

C

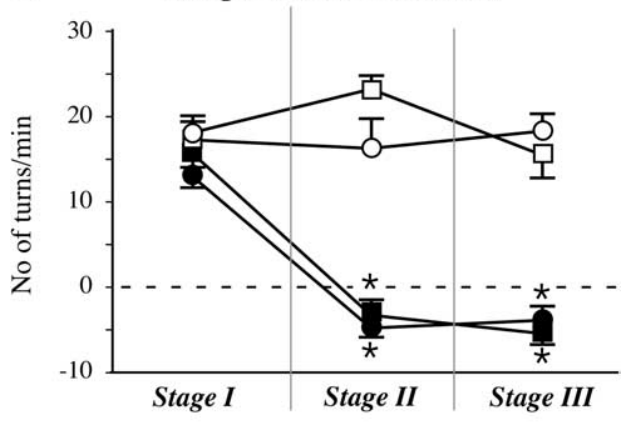

Figure 2. Behavioral tests. $\boldsymbol{A}-\boldsymbol{C}$, At every stage of the experiment, the animals were monitored in a battery of behavioral tests including the forelimb akinesia in the stepping test $(\boldsymbol{A})$, paw use bias in the cylinder test $(\boldsymbol{B})$, and rotational asymmetry after activation by amphetamine administration (C). The behavioral testing in stage 1 of the experiment was performed after the partial injury of the dopaminergic system (test session 1), where mainly the nigrostriatal projections were lesioned. This test session formed the basis for inclusion criteria in the experiment as well as the behavioral measures on which the groups were divided into two subgroups for stage 2. Basically, all animals that were included in the experiment had severe impairments in the stepping test and performed $<1$ step on average, representing $>90 \%$ loss of function $(\boldsymbol{A})$. Similarly, use of the left paw for wall touches in the cylinder test was reduced to $<15 \%(B)$, and a high rotational bias was seen in all animals in the amphetamine rotation (C). Stage 2 testing was performed 17-20 weeks after grafting, where the functional effects of the VM grafts were obvious in all three tests. Whereas in the stepping test the recovery was partial and the performance of the animals reached up to $\sim 50 \%$ of normal, in both the cylinder and amphetamineinduced rotation tests there was a full recovery. Behavioral tests in stage 3 were performed after the second lesion aimed at removing the remaining dopamine projections mainly to extrastriatal targets. The consequence of this on the behaviors that were initially improved by the graft was different. The benefits in the stepping test were dramatically reduced back to baseline values $(\boldsymbol{A})$, but only a partial but significant reduction was seen in the cylinder test. However, the amphetamine rotation test did not demonstrate any change from stage 2 . The dashed line in each panel represents the expected behavior of an intact animal. *Different from its control group; ${ }^{+}$different from striatal lesion (StrLes)-Graft-Sham group; "different from all other groups. MFBLes, MFB lesion. Error bars indicate SEM.

Extent of innervation provided by the grafted DA neurons

Surviving transplants were identified in all 16 animals that received VM cell suspension grafts. Graft-derived TH-positive fibers covered the areas of the striatum that were initially denervated by the intrastriatal 6-OHDA lesion. The restoration of striatal fiber density, as assessed by densitometry, was similar, $\sim 40-50 \%$ in both grafted groups (Fig. $3 Y$, compare $R, V)\left(F_{(3,34)}=\right.$ $8.72 ; p=0.0002)$. However, other areas normally innervated by the VTA neurons (i.e., frontal cortex, NAcb, and septum) did not receive any innervation from the grafts. Thus, in the doublelesioned animals, the innervation derived from the VM grafts was restricted to the caudate-putamen, whereas other forebrain DA targets remained denervated (Fig. 3, compare $Q-T, U-X$ ). Fiber density measurements in the NAcb confirmed this observation, indicating that the areas depleted by the second lesion were not innervated by the grafted DA neurons (Fig. $3 Z)\left(F_{(3,34)}=34.69\right.$; $p<0.0001)$. Importantly, however, the number of TH-positive cells in the transplants were similar in both grafted groups as shown in Figure 5, $A$ and $B$. The total number of TH-positive cells in the grafts were estimated to be $6865 \pm 1374$ and $5923 \pm 988$ in groups 3 and 4 , respectively (unpaired $t$ test, $p=0.58$ ), showing that the loss of behavioral benefits initially provided by the VM transplants in group 4 was not caused by a direct effect of the toxin on the established grafts after the second lesion.

\section{Discussion}

This study was designed to mimic the situation in a grafted PD patient when neurodegeneration in the mesencephalon progresses to include more and more of the DA neurons located outside the SN pars compacta, resulting in a progressively more widespread denervation of the forebrain target areas. Using a two-stage lesion paradigm, we investigated whether widening the denervating lesion to include projections to ventral striatal and nonstriatal forebrain areas would have a functional impact on the established intrastriatal VM grafts.

For this purpose, we first performed an intrastriatal 6-OHDA injection that was aimed to unilaterally remove large parts of the dopaminergic innervation of the caudate-putamen originating primarily from the $\mathrm{SN}$. This type of lesion results in an $\sim 80 \%$ loss of the TH-positive neurons in the SN pars compacta. These partially lesioned animals display severe impairments in motor performance tasks, as we have shown here by impaired paw use in the stepping and cylinder tests, and the amphetamine-induced turning response. The animals received grafts of fetal VM cell suspension distributed over five injection tracks to provide graft-derived TH-positive innervation of large areas in the caudate-putamen with good functional recovery in all three behavioral tests. In the third phase of the study, we extended the denervation of the host dopaminergic system by performing a second lesion by injecting 6-OHDA in the MFB in half of the animals, while the other half was subjected to a sham operation and followed as controls. The lesion resulted in degeneration of the remaining $20 \%$ of DA neurons in the $\mathrm{SN}$ and reduced the number of $\mathrm{TH}$-positive neurons in the VTA by $\sim 50 \%$. Consequently, the second lesion removed the remaining $\mathrm{TH}$-positive innervation in the rostromedial part of the head of caudate-putamen, as well as the projections to ventral striatum and NAcb, olfactory tubercle, and associated limbic and cortical forebrain areas. In animals for which a second lesion was performed, the graft-induced behavioral recovery in spontaneous tests, but not drug-induced rotation, was compromised, whereas the functional improvements remained stable in the respective control group. Thus, our results showed that the 


\section{A Group 1: StrLes-Sham-Sham}

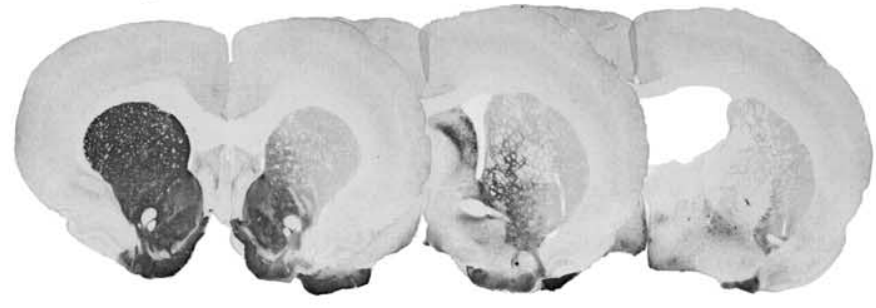

B Group 2: StrLes-Sham-MFBLes

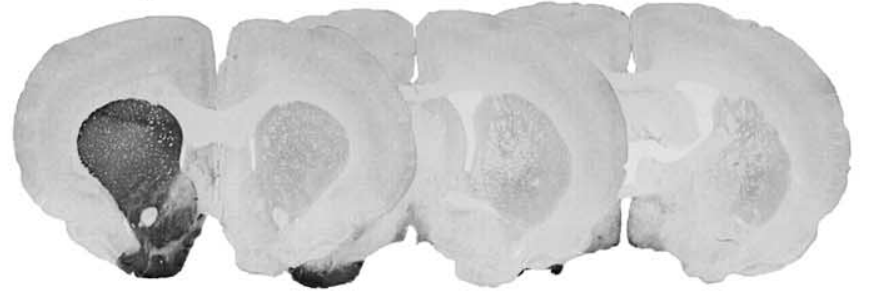

C Group 3: StrLes-Graft-Sham

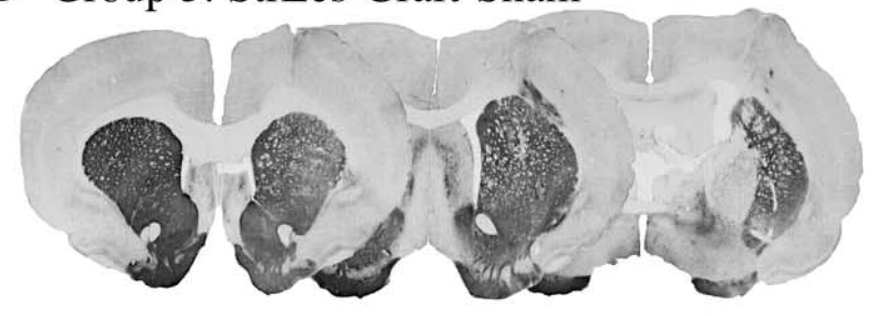

D Group 4: StrLes-Graft-MFBLes

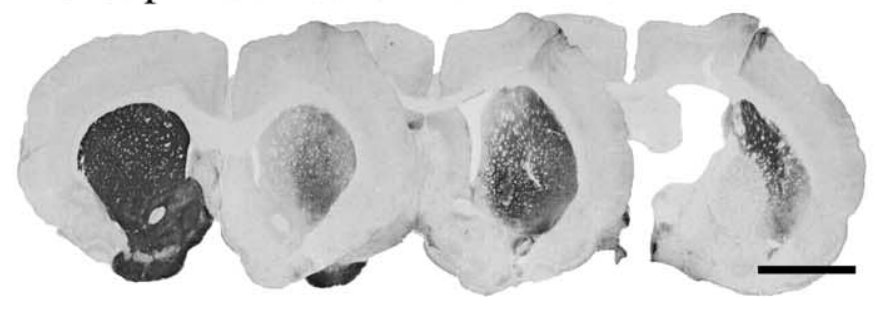

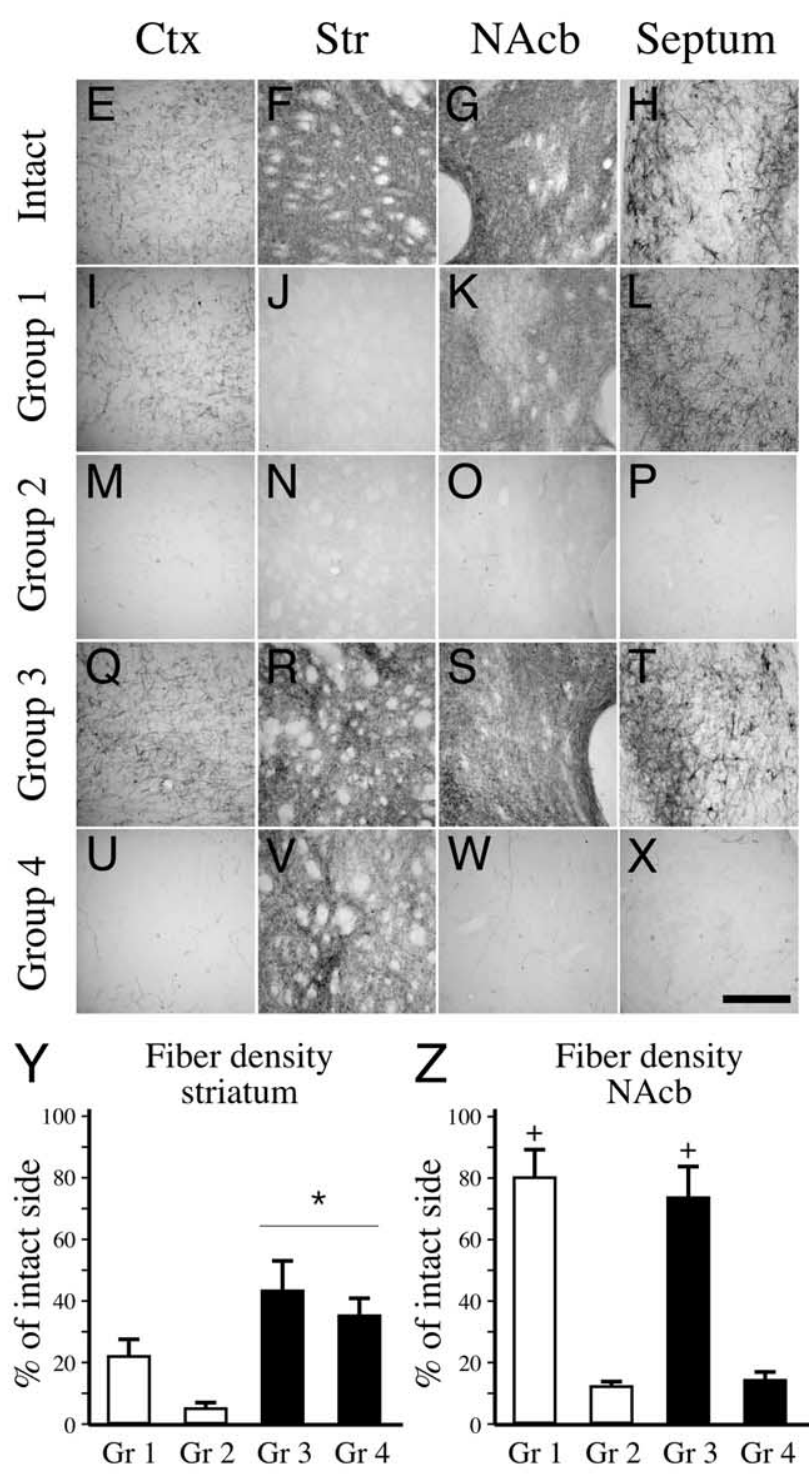

Figure 3. Ascending dopaminergic projections and fiber innervation provided by the graft. Four groups of animals were followed in this experiment. $\boldsymbol{A}$, $\boldsymbol{I}-\boldsymbol{L}$, In group 1 , the animals received a partial lesion depleting mainly the nigrostriatal projections to the dorsal and lateral striatum, leaving the mesocorticolimbic projections to the ventromedial striatum, nucleus accumbens, septum, and cortex intact. $\boldsymbol{B}, \boldsymbol{M}-\boldsymbol{P}$, Group 2 consisted of animals that received a second lesion, which depleted the mesocorticolimbic pathway removing nearly all of the midbrain dopamine input to the forebrain areas. $\boldsymbol{C}, \boldsymbol{D}, \mathbf{Q}-\boldsymbol{X}$, Groups $3(\boldsymbol{C}, \mathbf{Q}-\boldsymbol{T})$ and $4(\boldsymbol{D}, \boldsymbol{U}-\boldsymbol{X})$ were animals that received transplants of fetal ventral mesencephalic tissue in multiple tracts to provide widespread reinnervation in the striatum in partially and completely denervated animals, respectively. In group 3, reinnervation of the striatal areas was sufficient to reinstitute the dopamine input systems to the forebrain; however, although the graft-derived fibers were equally abundant in the striatum in group 4, degeneration of the mesocorticolimbic fibers left the graft-derived dopamine input isolated (compare $\mathbf{Q}-\boldsymbol{T}$ and $\boldsymbol{U}-\boldsymbol{X}$ with $\boldsymbol{E}-\boldsymbol{H}$ ). Semiquantitative analysis of total TH-positive fiber density was measured in the striatum (Y) and the nucleus accumbens (Z). (tx, Cortex; Str, striatum. Scale bars: (in $\boldsymbol{D}$ ) $\boldsymbol{A}-\boldsymbol{D}, 3 \mathrm{~mm}$; (in $\boldsymbol{X}) \boldsymbol{E}-\boldsymbol{X}, 250 \mu \mathrm{m}$. ${ }^{*}$ Different from nongrafted control groups; ${ }^{+}$different from the corresponding sham lesioned group. Error bars indicate SEM.

functional efficacy of intrastriatal VM grafts is to a large degree dependent on the extent of damage to the host DA system.

Loss of graft-derived functional benefits in MFB-lesioned animals can most directly be explained by the necessity for the integrity of a residual host DA system for appropriate (coordinated) functioning of the grafts with motor circuitries involving the striatal inputoutput pathways. We have found previously that the degree of functional recovery after grafting DA precursors into the denervated striatum well matches the functional performance expected for a corresponding level of TH-positive innervation of the host structure (i.e., comparable functional outcomes were observed as a result of similar levels of innervation provided either by the intrinsic DA neurons or by grafted DA neurons) (Kirik et al., 2001). Indeed, magni- tude of functional recovery seen in the grafted animals before the second lesion in the present study was almost identical to that seen in our previous experiments. In the double-lesioned animals, in contrast, the outcome was significantly compromised, seen as a clear worsening in forelimb use in the cylinder test and a complete loss of graft-induced functional benefit in the stepping test. Increasing the lesion severity had no impact on the turning response to amphetamine. The direction of turning in the amphetamine-induced rotation test is thought to be a consequence of an imbalance in DA release between the two dorsal striata (for review, see Dunnett and Robbins, 1992). This may explain the results we have observed in this study that in the grafted animals removal of the DA reinnervation in the NAcb does not lead to reinstitution of ipsilateral turning re- 

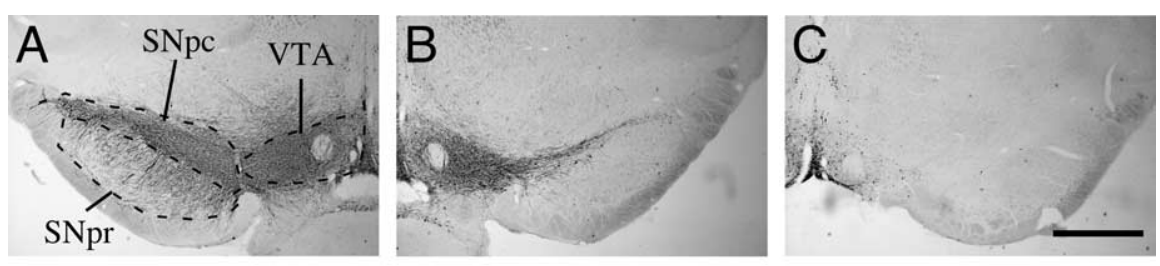

D $\mathrm{TH}+$ cells in $\mathrm{SN}$
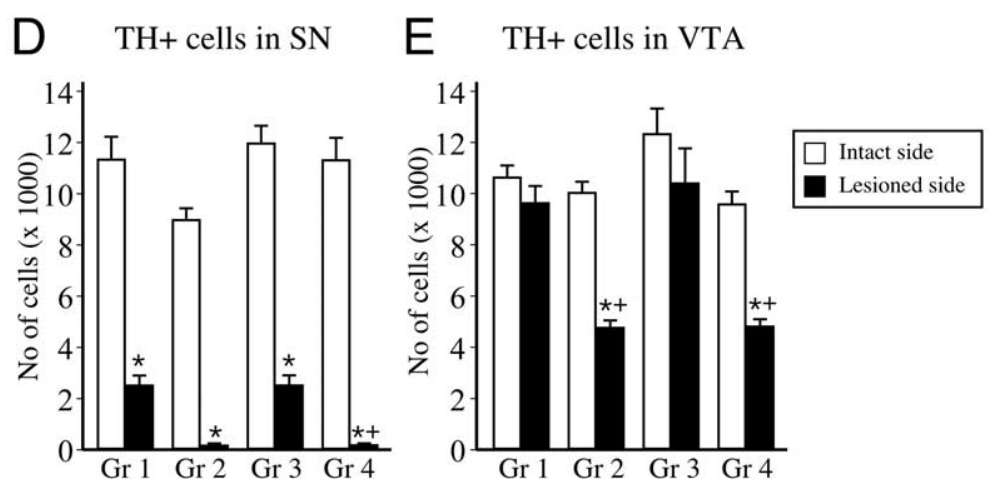

Figure 4. TH-positive cell numbers in the SN and the VTA that constitute the midbrain ascending projections to the forebrain. $A$, VTA neurons lie medially and project mainly to nonstriatal targets, whereas the laterally located SN cells preferentially innervate the striatum, as shown in a nonlesioned side of the brain. $\boldsymbol{B}$, Striatal injection of the 6-0HDA toxin leads to lesion of the striatal dopamine terminals and a retrograde degeneration of the SN cells, leaving most of the VTA neurons intact. $\boldsymbol{C}$, However, injection of the same toxin at the level of the medial forebrain bundle damages axons from both pathways and, thus, leads to a combined lesion affecting both projection systems. $\boldsymbol{D}, \boldsymbol{E}$, The total numbers of TH-positive cells present in each nuclei was quantified using computerized stereological estimation tools. We found that the striatal lesion in groups 1 and 3 lead to a comparable lesion of $\sim 75 \%$ in the SN. In these groups the VTA cell numbers were not affected. In the complete lesion animals (groups 2 and 4), however, not only the SN was completely depleted, but also the TH-positive cells in the VTA were reduced by $\sim 50 \%$. SNpc, Substantia nigra pars compacta; SNpr, substantia nigra pars reticulata. Scale bar: (in $\boldsymbol{C}$ ) $\boldsymbol{A}-\boldsymbol{C}, 1 \mathrm{~mm}$. *Different from intact side; ${ }^{+}$different from its control group. Error bars indicate SEM.
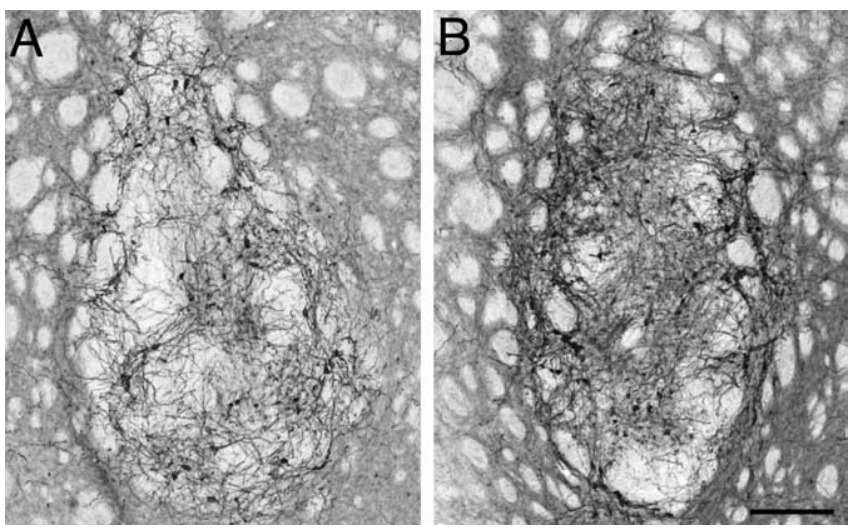

Figure 5. Survival of TH-positive cells in the striatum of animals grafted with ventral mesencephalic tissue. $\boldsymbol{A}, \boldsymbol{B}$, Two examples of graft deposits from a partially lesioned animal $(\boldsymbol{A})$ and a complete lesion animal $(\boldsymbol{B})$ are shown. We found that in both groups similar numbers of cells survived the transplantation ( $6865 \pm 1374$ and $5923 \pm 988$ cells in groups 3 and 4, respectively), showing that there was no effect of the second lesion on the grafted cells (unpaired $t$ test, $p=0.58)$. Scale bar, $200 \mu \mathrm{m}$.

sponse to amphetamine as seen in lesion control animals. Furthermore, it suggests that compensation of rotational asymmetry in the amphetamine-induced rotameter test may be used for rapid screening purposes to monitor survival and fiber outgrowth of grafted DA neurons, but is of limited value to assess their true functional impact in the rat PD model.

The level of functional recovery before the second MFB lesion was similar in both groups. Moreover, in the postmortem analysis, neither the number of TH-positive neurons in the grafts nor the extent of graft-derived TH-positive fiber outgrowth differed between the single-lesioned and the doublelesioned animals, indicating that the second injection of 6-OHDA into the host MFB did not have any adverse effect on the grafted DA neurons or their fiber outgrowths in the striatum. Whereas the DA denervation was strictly confined to the caudate-putamen in the single-lesioned rats, the extent of denervation in the double-lesioned animals included also the spared DA innervation of the rostromedial part of the head of the caudate-putamen, the ventral striatum and NAcb, olfactory tubercle, and the associated limbic and cortical forebrain areas.

In a clinical perspective, these data suggest that PD patients with DA cell loss restricted to the nigrostriatal projection system are most likely to respond well to striatal grafting compared with patients with more advanced disease in whom the DA projections to other areas of the forebrain are affected as well. The neurodegenerative process in PD brain is known to be rather heterogenous. Detailed histopathological analyses have revealed that in mild PD cases (with disease duration of $\sim 5$ years), neurodegeneration was most prominent (i.e., $\geq 80 \%$ cell loss) in the ventrolateral part of the SN. In contrast, the dorsal tier cell groups were lost only partially and later in the disease, with $\sim 40 \%$ cell survival in severe PD cases and disease duration of $\sim 25$ years (Fearnley and Lees, 1991). A similar close correlation between the loss of nigral DA neurons and disease duration has been shown by Damier et al. (1999). These authors also observed a marked variation in different midbrain cell groups from one patient to another. Cell death was invariably most pronounced in the ventral tier of the SN pars compacta (range of 75-95\% cell loss), whereas the dorsal tier neurons were less affected (range, 44-67\%) and the extent of cell loss appeared to be closely related to the duration of the disease. The extent of cell loss in other midbrain DA cell groups, however, did not show any correlation with disease duration. In this series, one patient with a 7 year disease history had severe cell loss in the medial cell group, whereas another with 25 year disease was almost unaffected (Damier et al., 1999). As a consequence, these cases in which cell loss is primarily limited to the ventral tier of the SN would be expected to have DA depletion mostly restricted to the putamen, which is invariably seen in all PD cases (Kish et al., 1988). However, DA denervation in other forebrain areas is likely to vary from patient to patient depending on the involvement of the DA cell groups in addition to the SN.

Together, these data suggest that the extent of DA denervation outside the restricted area of the caudate and putamen would have an impact on the functional outcome of DA neuron grafts in transplanted PD patients. This interpretation is supported by observations made by PET in grafted patients. In an analysis of nine patients who had received grafts of fetal midbrain DA neurons 1 to 2 years earlier, Piccini et al. (2005) have reported that the best functional outcome after transplantation was seen in subjects that exhibited no dopaminergic denervation in areas outside the 
grafted regions (as measured by ${ }^{18} \mathrm{~F}$-DOPA PET), either preoperatively or at the 1 or 2 years postoperative scans. In contrast, patients with no or modest graft-induced clinical benefit showed a marked reduction of ${ }^{18} \mathrm{~F}$-DOPA uptake in ventral striatal areas either before or after transplantation, indicating a more widespread DA neuron cell loss in these individuals (Piccini et al., 2005). Similarly, two recent National Institutes of Healthsponsored clinical trials reported that, as a group, older patients ( $>60$ years of age) and/or more advanced PD cases respond less well to grafts of fetal VM tissue placed in the putamen (Freed et al., 2001; Olanow et al., 2003). In future clinical trials, therefore, it may be important to make sure the patients selected for DA neuron transplantation have well preserved DA inputs to areas outside the grafted putamen (i.e., the areas innervated by the dorsal tier of the SN and the VTA). This could be accommodated by incorporation of high-resolution PET scans as part of the pregraft assessment criteria. However, because we currently do not have any means to predict the evolution of the disease prospectively on an individual basis, there will be an inherent uncertainty on whether some of the grafted patients may develop wider DA deficiencies compromising the impact of the otherwise functional grafts. Thus, for optimal clinical outcome in long term, it will probably be necessary to combine DA neuron grafting with measures to protect the remaining host DA system to maintain optimal graft-induced functional recovery in all grafted patients.

\section{References}

Brundin P, Pogarell O, Hagell P, Piccini P, Widner H, Schrag A, Kupsch A, Crabb L, Odin P, Gustavii B, Bjorklund A, Brooks DJ, Marsden CD, Oertel WH, Quinn NP, Rehncrona S, Lindvall O (2000) Bilateral caudate and putamen grafts of embryonic mesencephalic tissue treated with lazaroids in Parkinson's disease. Brain 123:1380-1390.

Cochen V, Ribeiro MJ, Nguyen JP, Gurruchaga JM, Villafane G, Loc'h C, Defer G, Samson Y, Peschanski M, Hantraye P, Cesaro P, Remy P (2003) Transplantation in Parkinson's disease: PET changes correlate with the amount of grafted tissue. Mov Disord 18:928-932.

Damier P, Hirsch EC, Agid Y, Graybiel AM (1999) The substantia nigra of the human brain. II. Patterns of loss of dopamine-containing neurons in Parkinson's disease. Brain 122:1437-1448.

Dunnett SB, Robbins TW (1992) The functional role of mesotelencephalic dopamine systems. Biol Rev Camb Philos Soc 67:491-518.

Fearnley JM, Lees AJ (1991) Ageing and Parkinson's disease: substantia nigra regional selectivity. Brain 114:2283-2301.

Freed CR, Greene PE, Breeze RE, Tsai WY, DuMouchel W, Kao R, Dillon S, Winfield H, Culver S, Trojanowski JQ, Eidelberg D, Fahn S (2001) Transplantation of embryonic dopamine neurons for severe Parkinson's disease. N Engl J Med 344:710-719.

Hagell P, Schrag A, Piccini P, Jahanshahi M, Brown R, Rehncrona S, Widner H, Brundin P, Rothwell JC, Odin P, Wenning GK, Morrish P, Gustavii B, Bjorklund A, Brooks DJ, Marsden CD, Quinn NP, Lindvall O (1999) Sequential bilateral transplantation in Parkinson's disease: effects of the second graft. Brain 122:1121-1132.

Hauser RA, Freeman TB, Snow BJ, Nauert M, Gauger L, Kordower JH, Olanow CW (1999) Long-term evaluation of bilateral fetal nigral transplantation in Parkinson disease. Arch Neurol 56:179-187.

Hirsch E, Graybiel AM, Agid YA (1988) Melanized dopaminergic neurons are differentially susceptible to degeneration in Parkinson's disease. Nature 334:345-348.

Kirik D, Rosenblad C, Bjorklund A (1998) Characterization of behavioral and neurodegenerative changes following partial lesions of the nigrostriatal dopamine system induced by intrastriatal 6-hydroxydopamine in the rat. Exp Neurol 152:259-277.

Kirik D, Winkler C, Bjorklund A (2001) Growth and functional efficacy of intrastriatal nigral transplants depend on the extent of nigrostriatal degeneration. J Neurosci 21:2889-2896.

Kish SJ, Shannak K, Hornykiewicz O (1988) Uneven pattern of dopamine loss in the striatum of patients with idiopathic Parkinson's disease. Pathophysiologic and clinical implications. N Engl J Med 318:876-880.

Kordower JH, Freeman TB, Snow BJ, Vingerhoets FJ, Mufson EJ, Sanberg PR, Hauser RA, Smith DA, Nauert GM, Perl DP, Olanow CW (1995) Neuropathological evidence of graft survival and striatal reinnervation after the transplantation of fetal mesencephalic tissue in a patient with Parkinson's disease. N Engl J Med 332:1118-1124.

Lindvall O, Brundin P, Widner H, Rehncrona S, Gustavii B, Frackowiak R, Leenders KL, Sawle G, Rothwell JC, Marsden CD, Bjorklund A (1990) Grafts of fetal dopamine neurons survive and improve motor function in Parkinson's disease. Science 247:574-577.

Mendez I, Dagher A, Hong M, Hebb A, Gaudet P, Law A, Weerasinghe S, King D, Desrosiers J, Darvesh S, Acorn T, Robertson H (2000) Enhancement of survival of stored dopaminergic cells and promotion of graft survival by exposure of human fetal nigral tissue to glial cell line-derived neurotrophic factor in patients with Parkinson's disease. Report of two cases and technical considerations. J Neurosurg 92:863-869.

Mendez I, Sanchez-Pernaute R, Cooper O, Vinuela A, Ferrari D, Bjorklund L, Dagher A, Isacson O (2005) Cell type analysis of functional fetal dopamine cell suspension transplants in the striatum and substantia nigra of patients with Parkinson's disease. Brain 128:1498-1510.

Nikkhah G, Bentlage C, Cunningham MG, Bjorklund A (1994) Intranigral fetal dopamine grafts induce behavioral compensation in the rat Parkinson model. J Neurosci 14:3449-3461.

Nyberg P, Nordberg A, Wester P, Winblad B (1983) Dopaminergic deficiency is more pronounced in putamen than in nucleus caudatus in Parkinson's disease. Neurochem Pathol 1:193-202.

Olanow CW, Goetz CG, Kordower JH, Stoessl AJ, Sossi V, Brin MF, Shannon KM, Nauert GM, Perl DP, Godbold J, Freeman TB (2003) A doubleblind controlled trial of bilateral fetal nigral transplantation in Parkinson's disease. Ann Neurol 54:403-414.

Olsson M, Nikkhah G, Bentlage C, Bjorklund A (1995) Forelimb akinesia in the rat Parkinson model: differential effects of dopamine agonists and nigral transplants as assessed by a new stepping test. J Neurosci 15:3863-3875.

Paxinos G, Watson C (1986) The rat brain in stereotaxic coordinates, Ed 2. San Diego: Academic.

Peschanski M, Defer G, N'Guyen JP, Ricolfi F, Monfort JC, Remy P, Geny C, Samson Y, Hantraye P, Jeny R, Gaston A, Kéravel Y, Degos JD, Cesaro P (1994) Bilateral motor improvement and alteration of L-dopa effect in two patients with Parkinson's disease following intrastriatal transplantation of foetal ventral mesencephalon. Brain 117:487-499.

Piccini P, Brooks DJ, Bjorklund A, Gunn RN, Grasby PM, Rimoldi O, Brundin P, Hagell P, Rehncrona S, Widner H, Lindvall O (1999) Dopamine release from nigral transplants visualized in vivo in a Parkinson's patient. Nat Neurosci 2:1137-1140.

Piccini P, Pavese N, Hagell P, Reimer J, Bjorklund A, Oertel WH, Quinn NP, Brooks DJ, Lindvall O (2005) Factors affecting the clinical outcome after neural transplantation in Parkinson's disease. Brain 128:2977-2986.

Schallert T, Tillerson J (1999) Intervention strategies for degeneration of dopamine neurons in parkinsonism: optimising behavioral assessment of outcome. In: Central nervous system diseases: innovative models of CNS disease: from molecule to therapy (Emerich DF, Dean III RL, Sandburg PR, eds), pp 131-151. Totowa, NJ: Humana.

Schallert T, Norton D, Jones TA (1992) A clinically relevant unilateral rat model of Parkinsonian akinesia. J Neural Transpl Plast 3:332-333.

Torres EM, Meldrum A, Kirik D, Dunnett SB (2006) An investigation of the problem of two-layered immunohistochemical staining in paraformaldehyde fixed sections. J Neurosci Methods 158:64-74.

Ungerstedt U, Arbuthnott G (1970) Quantitative recording of rotational behavior in rats after 6-hydroxydopamine lesions of the nigrostriatal dopamine system. Brain Res 24:485-493.

West MJ (1999) Stereological methods for estimating the total number of neurons and synapses: issues of precision and bias. Trends Neurosci 22:51-61.

Winkler C, Bentlage C, Nikkhah G, Samii M, Bjorklund A (1999) Intranigral transplants of GABA-rich striatal tissue induce behavioral recovery in the rat Parkinson model and promote the effects obtained by intrastriatal dopaminergic transplants. Exp Neurol 155:165-186.

Winkler C, Kirik D, Bjorklund A (2005) Cell transplantation in Parkinson's disease: how can we make it work? Trends Neurosci 28:86-92. 